\title{
Management of vascular uterine lesions associated with persistent low-level human chorionic gonadotrophin
}

\author{
Kate L Darlow, Andrew W Horne, Hilary O D Critchley, Jane Walker, W Colin Duncan
}

\begin{abstract}
Background and methodology Vascular lesions of the uterus are rare but pose difficult management decisions. We reviewed the cases of four patients who presented with vascular uterine lesions following early pregnancy loss at the Royal Infirmary of Edinburgh, Edinburgh, UK in 2006.
\end{abstract}

Results All four patients had signs on colour Doppler imaging consistent with arteriovenous malformations and elevated serum human chorionic gonadotrophin (hCG) levels. In each case, once the hCG level had returned to normal the uterine lesions resolved. The first case required therapeutic embolisation but the subsequent patients were successfully managed conservatively.

Conclusions Most uterine vascular lesions are attributed to arteriovenous malformations but those associated with pregnancy represent subinvolution of the placental bed and can be managed conservatively with monitoring of hCG concentrations.

Keywords embolisation, haemorrhage, human chorionic gonadotrophin (hCG), miscarriage, vascular malformation

J Fam Plann Reprod Health Care 2008; 34(2): 118-120

(Accepted 14 January 2008)

\section{Introduction}

Vascular lesions are a rare finding when imaging is performed for women who present with heavy vaginal bleeding and they pose difficult clinical management dilemmas. They can represent a true arteriovenous malformation (AVM), a haemangioma, gestational trophoblastic disease or retained products of conception. An AVM may be congenital, or acquired through pelvic surgery, infection, trophoblastic disease, cervical or endometrial malignancy and trauma including curettage. 1,2

Colour Doppler imaging is the preferred method of diagnosis, with angiography reserved for patients requiring therapeutic embolisation. 2,3 The recommended diagnostic signs for an AVM are based on the presence of a hypervascular lesion with turbulent flow within the myometrium. ${ }^{1}$ However, these Doppler findings may also be observed in gestational trophoblastic disease and with retained products of conception (RPOC). ${ }^{1}$

Management of such lesions ranges from a conservative approach to invasive procedures including embolisation or hysterectomy.

\section{Report of cases}

Over a 1-year period, six patients with a vascular uterine lesion following early pregnancy failure in the first trimester were identified in the Simpson Centre for Reproductive Health at the Royal Infirmary of Edinburgh, Edinburgh, UK. We searched the available literature for advice on management but found a lack of clear guidelines for diagnosis or treatment. This prompted us to review the

Simpson Centre for Reproductive Health, Royal Infirmary of Edinburgh, Edinburgh, UK

Kate L Darlow, MBChB, Specialist Registrar

Department of Reproductive and Developmental Sciences, University of Edinburgh, UK

Andrew W Horne, PhD, MRCOG, Principal Lecturer

Hilary O D Critchley, MD, FRCOG, Professor of Reproductive

Medicine

W Colin Duncan, MD, MRCOG, Consultant

Department of Radiology, Royal Infirmary of Edinburgh, Edinburgh, UK

Jane Walker, MBChB, FRCR, Consultant Radiologist

Correspondence to: Dr Kate Darlow, Department of Obstetrics and Gynaecology, St John's Hospital, Howden, Livingston, West Lothian EH54 6PP, UK. E-mail: katedarlow@doctors.org.uk

\section{Key message points}

- Conservative management of vascular uterine lesions is appropriate when they are associated with elevated human chorionic gonadotrophin.

- If such lesions are associated with very heavy bleeding, operative interference may be avoided by therapeutic embolisation.

case records of the first four patients, which then influenced our clinical decisions for subsequent patients.

\section{Case 1}

The first case was a 36-year-old nulliparous woman who presented as an emergency with heavy vaginal bleeding, 8 weeks' amenorrhoea and a positive pregnancy test. She had a background of an irregular menstrual cycle since stopping the oral contraceptive pill 18 months previously.

A pelvic ultrasound scan showed no evidence of an intrauterine pregnancy and the myometrium was of a normal texture. The patient's serum human chorionic gonadotrophin (hCG) at that time was $81 \mathrm{IU} / 1$. The impression was that the patient had had a complete miscarriage although an ectopic pregnancy could not be excluded. She was therefore asked to return for a repeat serum hCG 48 hours later. The repeat hCG was $46 \mathrm{IU} / 1$, and 1 week later showed a slower fall than expected to $36 \mathrm{IU} / \mathrm{l}$.

One week subsequently she returned with lower abdominal pain, ongoing light vaginal bleeding and a lowgrade pyrexia. A repeat hCG was $22 \mathrm{IU} / 1$. She was admitted overnight and treated with intravenous antibiotics for suspected endometritis. After a further 10 days she returned with a history of much heavier vaginal bleeding and crampy abdominal pain. Her serum hCG was still 13 IU/l. A transvaginal ultrasound scan (TVS) showed an $8 \times 6 \times 11$ $\mathrm{mm}$ area of mixed echogenicity in the endometrium of the uterine fundus. In addition, the anterior myometrial wall contained a highly vascular area, with both arterial and venous flow on Doppler examination (Figure 1a). These appearances were suggestive of minimal RPOC and a possible uterine vascular malformation.

The management plan was for the patient to have a repeat TVS in 1 month. However, she returned as an emergency 3 weeks later, with further very heavy bleeding. Her haemoglobin fell from 119 to $95 \mathrm{~g} / \mathrm{l}$ and her serum hCG was still raised at $8 \mathrm{IU} / \mathrm{l}$. A repeat TVS showed increasing vascular sinuses in the uterine wall. An AVM 

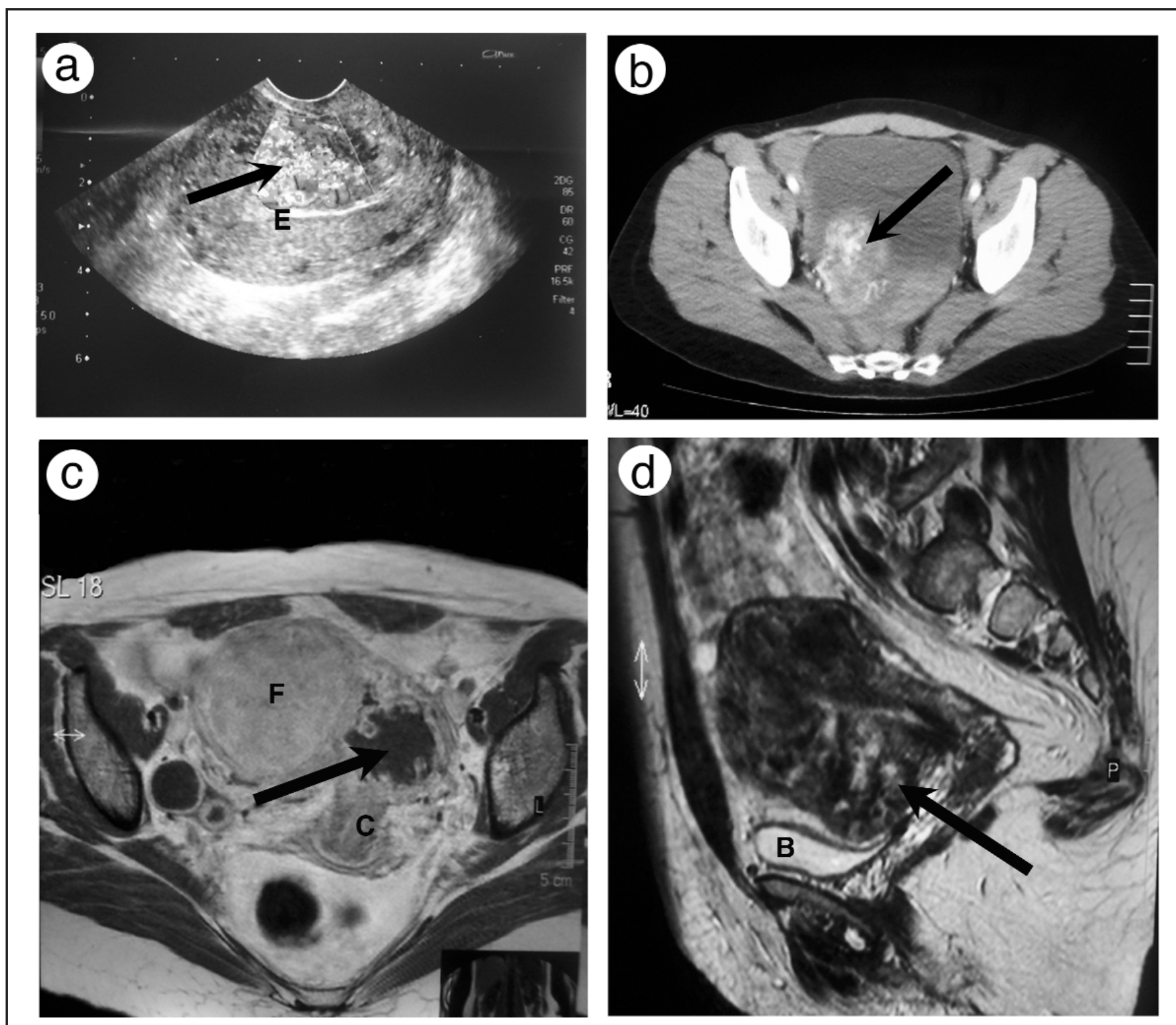

Figure 1 Imaging in vascular lesions. Case 1. (a) Transvaginal ultrasound scan showing a largely thin endometrial echo (E) with a highly vascular area on colour flow Doppler (arrow) on the anterior uterine wall. (b) Computed tomography scan with contrast shows a brightly enhancing vascular serpiginous structure (arrow) extending from the uterine wall with extravasation into the uterine cavity. Case 2. (c) Magnetic resonance imaging (MRI) scan of fibroid (F) and abnormal vascular area (arrow) above cervix (C). (d) Vascular enhanced MRI scan of uterus with increased vascularity throughout the uterine wall (arrow) distorting the bladder (B)

was suspected and the patient was therefore taken for urgent radiological intervention. A computed tomography (CT) scan showed marked enhancement of the anterior uterine wall with contrast extravasation into the uterine cavity (Figure 1b). This was in keeping with active bleeding from a uterine vascular malformation. However, the lack of early venous filling and the appearance of the vessels during the angiography were not typical of AVM. She went on to undergo bilateral uterine artery embolisation, which was successful in stemming the heavy vaginal bleeding.

Four months following embolisation the patient was asymptomatic, having re-established a normal menstrual cycle. Her hCG was normal at $<5 \mathrm{IU} / 1$ and a follow-up magnetic resonance imaging (MRI) scan showed a normal uterus.

\section{Case 2}

The second case was a 40-year-old woman who presented with a 3-year history of secondary infertility. She had had one early miscarriage requiring surgical evacuation and a Caesarean section at term for transverse lie. She underwent routine fertility investigations, including a TVS. The only abnormality found was a $6 \mathrm{~cm}$ intramural fibroid at the uterine fundus, which did not distort the cavity. She then became pregnant spontaneously but approximately 7 weeks from her last menstrual period she presented as an emergency with vaginal bleeding. TVS demonstrated fetal demise and she underwent an uncomplicated surgical evacuation with minimal blood loss.

One month later she was reviewed in our infertility clinic and a TVS was performed to assess the fibroid. This remained unchanged, but a $3.7 \times 5.3 \mathrm{~cm}$ area of mixed echogenicity, with an increased vascular signal, was noted near the cervix. At this time the patient was asymptomatic. However, her serum hCG was found to be elevated at 686 IU/1. The possibility of a hydatidiform mole was raised and consequently her hCG levels were monitored. As these fell, this diagnosis was thought unlikely and a plan was made for conservative management. Over the following month 
the patient remained asymptomatic and her hCG declined slowly to $90 \mathrm{IU} / \mathrm{l}$.

Before her next appointment, the patient presented as an emergency with heavy vaginal bleeding. Her serum hCG at this stage was slightly higher at 114 IU/1. A TVS showed a $6 \mathrm{~cm}$ mass of mixed echogenicity, which appeared to extend from the uterine cavity anteriorly into and through the myometrium. It was located low in the body of the uterus, in the region of the previous Caesarean section scar. Colour Doppler imaging revealed the mass to be highly vascular with low resistance flow. The cause of the bleeding at this time was uncertain but the differential diagnosis included RPOC, molar change and vascular malformation and it was therefore important to establish a tissue diagnosis.

Hysteroscopy showed a large red polyp-like structure, in continuity with the uterine wall, extending into the cavity. Minimal bleeding occurred during the procedure. A careful biopsy was obtained. This showed inflamed and necrotic decidua, but no chorionic villi or trophoblast.

As a result of the findings at hysteroscopy an MRI scan was performed (Figure 1c), which confirmed the presence of a vascular lesion close to the cervix that crossed the myometrium and distorted the bladder (Figure 1d). After discussion between gynaecology and radiology colleagues it was decided that a conservative approach was the best line of management. Over the following weeks the patient was monitored as an outpatient. Her hCG levels gradually fell to normal and her menstrual cycle returned to a normal pattern. A repeat TVS at 3 months showed a normal uterine cavity with complete resolution of the vascular lesion.

\section{Cases 3 and 4}

The third patient was a 26-year-old woman who underwent surgical management of an incomplete miscarriage at 12 weeks and the fourth was a 24-year-old woman who had undergone surgical termination of pregnancy at 10 weeks. Both women re-presented several weeks later with heavy vaginal bleeding and were found to have vascular myometrial lesions on Colour Doppler imaging associated with elevated hCG levels. The vascular lesion in the fourth patient increased in size (from $2.2 \times 2.7 \times 2.7 \mathrm{~cm}$ to $4.7 \times$ $4.1 \times 3.9 \mathrm{~cm})$ and became more pronounced over the next month with marked vascular sinuses. Both patients were managed conservatively, and within 2 to 3 months their hCG levels had returned to normal and repeat TVSs showed entirely normal uterine morphology.

\section{Discussion}

This case series describes the management of four patients found to have vascular lesions of the uterus after spontaneous miscarriage or first-trimester termination of pregnancy.

With each patient, the colour Doppler imaging findings on TVS were consistent with the recognised signs for an AVM. However, angiography performed in the first case did not show early venous contrast filling, which is the cardinal sign of AVM. ${ }^{3}$ Additionally, the spontaneous regression of the vascular lesions in our series is not typical of true AVMs.
Histopathological investigations have shown that AVMs occur as a result of errors in morphogenesis. They are composed of stable endothelial cells, which therefore do not show spontaneous regression. ${ }^{4}$

The presence of uterine vascular malformations following pregnancy has been reported by Timmerman et $a l$. who felt that the condition may represent subinvolution of the placental bed. ${ }^{3}$ Similarly, our impression is that although the ultrasound imaging of our case series mimicked true AVMs, these lesions do represent subinvolution of retained placental tissue. By tracking the patients' hCG levels, which were all found to be elevated and which resolved slowly as the vascular lesions regressed, we have been able to support this hypothesis. It is not known whether the trophoblast was karyotypically normal in these cases, but we do note the association with miscarriage suggesting that there may be a link to abnormal placentation or trophoblast function.

In the case report by Kido et al..$^{5}$ a vascular lesion was noted within the uterus 6 weeks after surgical termination of pregnancy. A hysterectomy was performed for a suspected AVM. However, pathology revealed only necrotic RPOC. These authors' hypothesis was that necrosis of the chorionic villi left vessels with arteriovenous fistulas in the retained placental tissue. It was only noted retrospectively that serum hCG levels were elevated and returned to normal postoperatively. As with the first case in our series, intervention was necessary in view of the volume of bleeding. However, if this were not a factor, it is likely that the vascular lesion would have resolved spontaneously.

\section{Conclusion}

It is appropriate that vascular uterine lesions following early pregnancy loss are managed conservatively with hCG monitoring, with the exception of patients who bleed heavily and who are therefore at risk of vascular compromise, who may be managed with therapeutic embolisation.

Statements on funding and competing interests

Funding None identified.

Competing interests None identified.

\section{References}

1 Mungen E. Vascular abnormalities of the uterus: have we recently over-diagnosed them? Ultrasound Obstet Gynecol 2003; 21: 529-531.

2 Timmerman D, Van den Bosch T, Peeraer K, Debrouwere E, Van Schoubroeck D, Stockx L, et al. Vascular malformations in the uterus: ultrasonographic diagnosis and conservative management. Eur J Obstet Gynecol Reprod Biol 2000; 92: 171-178.

3 Timmerman D, Wauters J, Van Calenbergh S, Van Schoubroeck D, Maleux G, Van Den Bosch T, et al. Color Doppler imaging is a valuable tool for the diagnosis and management of uterine vascular malformations. Ultrasound Obstet Gynecol 2003; 21: 570-577.

4 Mulliken JB, Glowacki J. Hemangiomas and vascular malformations in infants and children: a classification based on endothelial characteristics. Plast Reconstr Surg 1982; 69: 412-422.

5 Kido A, Togashi K, Koyama T, Ito H, Tatsumi K, Fujii S, et al. Retained products of conception masquerading as acquired arteriovenous malformation. J Comput Assist Tomogr 2003; 27: 88-92.

\section{PEER REVIEWERS}

If you have a special interest in one or more of the topics covered by the Journal and have some time available to peer review occasional papers in your own area(s) of expertise then perhaps you might be interested in joining the Journal's team of peer reviewers? In common with the majority of other academic journals, peer reviewers offer their services on a voluntary basis; however, if you are a member of the Faculty of Sexual and Reproductive Healthcare then each completed review counts for two (2) CME points. For further information please contact the Journal Editorial Office at journal@fsrh.org. 